\title{
COMPOSTOS ORGÂNICOS COMO SUBSTRATOS NA FORMAÇÃO DE MUDAS DE IPÊ-AMARELO (Tabebuia chrysotricha( Mart. Ex. Dc.) standl) IRRIGADAS COM ÁGUA RESIDUÁRIA
}

\author{
REGINALDO I. MURAISHI ${ }^{1}$, JOÃO A. GALBIATTI' ${ }^{2}$, FABIO O. DE NOBILE ${ }^{3}$, \\ JOSÉ C. BARBOSA ${ }^{4}$
}

\begin{abstract}
RESUMO: A necessidade de estudar a utilização dos resíduos de podas de árvores é de grande importância ambiental para solucionar os problemas de resíduos sólidos existentes nas áreas urbanas junto com os resíduos de lixos domésticos. $O$ estudo destes materiais foi avaliado com o desenvolvimento de mudas de ipê-amarelo (Tabebuia Chrysotricha (Mart. Ex. Dc.) sandl) em diferentes misturas de substratos e tipos de água para irrigação. O experimento foi instalado no Departamento de Engenharia Rural da Faculdade de Ciências Agrárias e Veterinárias - UNESP, Câmpus de Jaboticabal. Foram realizados dois experimentos, avaliados juntamente com delineamento experimental em blocos casualizados, de oito substratos, duas qualidades de águas e quatro repetições, totalizando 64 parcelas. Cada parcela foi composta por 30 plantas (cinco linhas de seis plantas), sendo consideradas como úteis as três linhas de quatro plantas centrais da parcela. Foram testados oito substratos, resultantes da combinação de substrato comercial, composto de lixo e composto de poda de árvores com dois tipos de água de irrigação (água potável e residuária) e quatro repetições. Para acompanhar o desenvolvimento das mudas de ipê-amarelo, foram avaliados a altura da parte aérea das plantas $(\mathrm{H})$ e o diâmetro do colmo (D). As características foram avaliadas aos 21; 42; 63 e 84 dias após a emergência. Da análise dos resultados, possibilitou-se concluir que os substratos estudados promoveram diferenças significativas para a altura média das plantas e o diâmetro de colmo, em todos os períodos de avaliação. Os substratos 4 e 5 e a água residuária apresentaram os melhores resultados no desenvolvimento das mudas de ipê-amarelo.
\end{abstract}

PALAVRAS-CHAVE: Altura de plantas, diâmetro de caule, qualidade da água.

\section{ORGANIC COMPOUND AS SUBSTRATE IN THE FORMATION OF THE YELLOW-IPE (Tabebuia chrysotricha (Mart. Ex DC.) Standl) SEDLINGS IRRIGATED WITH RESIDUARY WATER}

\begin{abstract}
The need to study the use of waste of tree pruning is of great importance to solve the environmental problems of solid waste in the urban areas along with the household waste. The study of these materials was evaluated with the development of seedlings of yellow- ipe (Tabebuia Chrysotricha (Mart. Ex DC.) Sandl) in different types of substrate mixtures and water for irrigation. The experiment was installed at the Department of Rural Engineering in the Faculty of Agricultural Sciences and Veterinary - UNESP, Jaboticabal Campus, in a randomized block design, with 8 substrates, 2 qualities of water and 4 repetitions, totaling 64 plots. Each plot was composed of 30 plants (5 lines of 6 plants), being regarded as useful, the 3 lines of 4 plants of the central plot. Eight substrates were tested, resulting from the combination of commercial substrate, composed of garbage and of trees pruning with 2 types of irrigation water (clean water and residuary) with 4 repetitions. To track the development of the yellow-ipe seedlings, were evaluated the height of the shoots $(\mathrm{H})$ and the diameter of the stem (D). The characteristics were evaluated at 21, 42, 63 and 84 days after the emergency. The analysis of the results allowed to conclude that the substrates studied promoted significant difference in average height and diameter of the stems in all periods of assessment. The substrate 4, 5 and the residuary water showed the best results in the development of the yellow-ipe seedlings.
\end{abstract}

KEYWORDS: Height of plants, stem diameter, water quality.

\footnotetext{
${ }^{1}$ Professor das disciplinas Ecologia Aplicada e Gestão de Recursos Naturais, UNIFEB, Barretos - SP, itimuraishi@hotmail.com.

${ }_{2}^{2}$ Prof. Dr., Faculdade de Ciências Agrárias e Veterinárias, UNESP, Câmpus de Jaboticabal - SP, galbi@ @cav.unesp.br.

${ }^{3}$ Professor de Solos, Adubos e Adubação, UNIFEB, Barretos - SP, fonobile@ feb.br.

${ }^{4}$ Professor Titular, Departamento de Ciências Exatas, FCAV/UNESP, Jaboticabal - SP. 


\section{INTRODUÇÃO}

Segundo dados do IBGE (2002), 59\% dos municípios dispõem os resíduos coletados inadequadamente em lixões ou mesmo em aterros controlados. Os problemas decorrentes dessas atitudes são bastante conhecidos, como transmissões de doenças, impactos ambientais e sociais. A compostagem, aliada à reciclagem dos materiais inertes, pode reduzir significativamente a quantidade de resíduos a ser disposta no ambiente (GROSSI, 1993), pois quase toda a parte orgânica do lixo é aproveitada.

Uma área de aplicação que não acarreta problemas de saúde humana é a utilização de tais adubos orgânicos como componentes de substrato para produção de mudas de espécies ornamentais e de arborização urbana. Neste sentido, SANDERSON (1980) salienta que a utilização de composto seria ideal para produção de plantas ornamentais, uma vez que estas não são utilizadas na alimentação humana e, portanto, não oferecem riscos à saúde. A importância ambiental e social da adequada produção de composto de lixo urbano, bem como a sua aplicação agronômica como fonte de matéria orgânica e de nutrientes para as culturas, é documentada por vários autores (OLIVEIRA et al. 2002).

O composto de lixo também possui vários micronutrientes, como Zinco, Manganês e Cobre, que podem ser liberados para as plantas, reduzindo ou mesmo substituindo o uso de fertilizante. Entretanto, o composto de má qualidade, isto é, aquele originário de lixo indevidamente coletado e separado, pode conter metais potencialmente tóxicos, como chumbo, crômio, cádmio e níquel, que uma vez adicionados ao solo podem ser absorvidos pelas plantas, entrando assim na cadeia alimentar.

No setor urbano, diariamente, são feitos cortes de galhos e árvores, chegando a vários caminhões por dia. Após a seleção, geralmente, o material fino e as folhas são descartados, e aqueles de maior diâmetro são armazenados e utilizados como lenha. A pouca utilização desse material talvez esteja na falta de conhecimento das opções de uso, e a compostagem pode ser uma alternativa. Segundo BENITO et al. (2005), estudos de estágios da maturidade e da estabilidade de resíduos de poda na compostagem mostram que a atividade da enzima desidrogenase e a evolução do $\mathrm{CO}_{2}$ podem ser usadas como bom indicador em processo de compostagem de poda de árvores.

Pensando na reutilização futura de efluentes, a atividade florestal, por suas peculiaridades, apresenta-se como uma alternativa promissora, principalmente por não envolver produção de alimentos para consumo humano nem riscos à saúde (CROMER, 1980). Além disto, por ser realizada em larga escala, tem a potencialidade de consumir grande volume de efluentes. Segundo LUCENA et al. (2006), a utilização de águas residuárias pré-tratadas na irrigação de mudas de essências florestais poderá representar uma alternativa promissora na produção de mudas de boa qualidade, com baixo custo e, sobretudo, criando opção para o uso dessa água. A utilização da água residuária é uma opção vantajosa, não apenas pelo aporte de nutrientes que contêm estas águas, mas, sobretudo, por contribuir com a preservação dos corpos de água.

AUGUSTO et al. (2003) sugerem que a água residuária pode ser utilizada na fertirrigação de viveiros para a produção das espécies de Croton floribundus (capixingui) e Copaifera langsdorffii (Copaíba), pois todas as plantas se mostraram vigorosas, com bom desenvolvimento, sem mortalidade, deficiência ou toxidez. Diante do exposto, o objetivo deste trabalho foi estudar o comportamento de mudas de ipê-amarelo (Tabebuia chrysotricha (Mart. Ex. Dc.) stand), desenvolvidas em substratos formados pela associação entre diferentes doses de compostos de lixo doméstico, composto de poda de árvore e substrato comercial, em condição de irrigação com água potável e residuária.

\section{MATERIAL E MÉTODOS}

O experimento foi desenvolvido no Departamento de Engenharia Rural da Faculdade de Ciências Agrárias e Veterinárias - UNESP, Câmpus de Jaboticabal - SP. A classificação climática para a região, segundo Köeppen, é do tipo Aw, ou seja, subtropical úmido com estiagem no inverno. 
A precipitação e as temperaturas médias anuais situam-se próximas de $1.400 \mathrm{~mm}$ e $21^{\circ} \mathrm{C}$, respectivamente. $\mathrm{O}$ experimento foi desenvolvido em ambiente protegido com plástico leitoso, com $50 \%$ de retenção da luz e sombrite. Foram realizados dois experimentos, e o delineamento experimental adotado foi o em blocos casualizados, com oito substratos, duas qualidades de águas e quatro repetições, totalizando 64 parcelas. Cada parcela foi composta por 30 plantas (cinco linhas de seis plantas), sendo consideradas como úteis as três linhas de quatro plantas centrais da parcela. Os substratos avaliados, resultantes da combinação de substrato comercial, composto de lixo e composto de poda de árvores (Tabela 1), foram submetidos à análise, em conjunto dos dois experimentos.

TABELA 1. Percentual dos materiais na composição volumétrica dos substratos utilizados para produção de mudas de Tabebuia chrysotricha (ipê-amarelo). Percentage of material in the volumetric composition of substrates used for seedling production of Tabebuia chrysotricha (yellow-ipe).

\begin{tabular}{cccc}
\hline Substratos & Substrato Comercial & Composto de Lixo & Composto de Poda \\
\hline 1 & 100 & 0 & 0 \\
2 & 0 & 100 & 0 \\
3 & 0 & 5 & 95 \\
4 & 0 & 10 & 90 \\
5 & 0 & 20 & 80 \\
6 & 0 & 40 & 60 \\
7 & 0 & 60 & 40 \\
8 & 0 & 0 & 100 \\
\hline
\end{tabular}

A planta utilizada foi da Tabebuia chrysotricha (Mart. ex. Dc.) standl, devido à importância da espécie como madeira de lei, largamente utilizada em paisagismo, arborização urbana, além de ser árvore símbolo do Brasil e presente em todo o território nacional.

As sementes para a formação de muda de Tabebuia chrysotricha (ipê-amarelo) foram obtidas junto ao viveiro (horto) de mudas ornamentais e florestais da FCAV/UNESP - Jaboticabal - SP. Foram utilizados tubetes de polietileno com capacidade de $300 \mathrm{~cm}^{3}$ de volume, preenchidos com substratos descritos na Tabela 1. O composto de poda de árvore foi obtido na cidade de Guaíra - SP, e o composto de lixo doméstico na cidade de São José do Rio Preto - SP. As misturas dos substratos foram realizadas manualmente, conforme os tratamentos indicados na Tabela 1, cujas características química e física foram avaliadas no laboratório da ESALQ/USP - Piracicaba - SP (Tabela 2).

TABELA 2. Dados das características químicas e físicas dos substratos testados. Chemical and physical characteristics of substrates tested.*

\begin{tabular}{lccc}
\hline Determinações & Substrato Comercial & Composto de Lixo & Composto de Poda \\
\hline pH em CaCl2 $0,01 \mathrm{M}$ & 5,2 & 7,8 & 7,1 \\
Densidade $\left(\mathrm{g} / \mathrm{m}^{3}\right)$ & 0,64 & 0,58 & 0,68 \\
Carbono total $($ orgânico e mineral $)(\%)$ & 15,68 & 21,16 & 11,37 \\
Nitrogênio total $(\%)$ & 0,49 & 1,44 & 0,97 \\
Fósforo $\left(\mathrm{P}_{2} 0_{5}\right)$ total $(\%)$ & 0,06 & 0,60 & 0,19 \\
Potássio $\left(\mathrm{K}_{2} 0\right)$ total $(\%)$ & 0,15 & 0,38 & 0,80 \\
Cálcio $(\mathrm{Ca})$ total $(\%)$ & 1,27 & 4,21 & 1,84 \\
Magnésio $(\mathrm{Mg})$ total $(\%)$ & 0,62 & 0,27 & 0,18 \\
Relação C/N (C total e N total) & $32 / 1$ & $15 / 1$ & $12 / 1$ \\
Relação C/N (C orgânico e N total) & $32 / 1$ & $14 / 1$ & $12 / 1$ \\
\hline
\end{tabular}

*Análises química e física foram realizadas conforme metodologia do LABORATORIO NACIONAL DE REFERÊNCIA VEGETAL (1988).

A irrigação foi realizada com água residuária obtida no córrego Jaboticabal, próximo da estação de esgoto de Jaboticabal-SP, e a água potável obtida no local do experimento, proveniente 
de poço artesiano da FCAV/UNESP (Tabela 3). Em cada tubete, foram semeadas duas sementes de Tabebuia chrysotricha, sendo efetuado o raleio 30 dias após a emergência, deixando-se uma planta por tubete. As análises químicas constaram teores de macro e micronutrientes $(\mathrm{N}, \mathrm{P}, \mathrm{K}, \mathrm{Ca}, \mathrm{Mg}, \mathrm{S}$, $\mathrm{B}, \mathrm{Cu}, \mathrm{Fe}, \mathrm{Mn}, \mathrm{Zn}), \mathrm{Na}$, metais pesados ( $\mathrm{Cd}, \mathrm{Pb}, \mathrm{Ni}, \mathrm{Cr}$ ), conforme metodologia descrita em $\mathrm{HACH}$ (1996) (Tabela 3).

TABELA 3. Características químicas das águas testadas. Chemical characteristics of the tested waters.*

\begin{tabular}{|c|c|c|c|c|c|c|c|c|c|}
\hline & $\begin{array}{c}\mathrm{pH} \\
\mathrm{CaCl}_{2}\end{array}$ & $\begin{array}{l}\text { C.E. } \\
\mathrm{dS} \mathrm{m}^{-1}\end{array}$ & $\mathrm{~N}-\mathrm{NH}_{3}$ & $\mathrm{~N}-\mathrm{NO}_{2}$ & $\mathrm{~N}-\mathrm{NO}_{3}$ & $\begin{array}{r}\mathrm{PO}_{4} \\
\mathrm{mg} \mathrm{L}^{-1}-\end{array}$ & $\mathrm{K}$ & $\mathrm{Ca}$ & $\mathrm{Mg}$ \\
\hline Água Potável & 6,3 & 123 & 0,292 & 0,007 & 0,019 & 0,353 & 0 & 0,06 & 0 \\
\hline \multirow[t]{3}{*}{ Água Residuária } & 6,9 & 282 & 0,870 & 0,006 & 0,013 & 0,705 & 0 & 0,05 & 0 \\
\hline & $\mathrm{Na}$ & $\mathrm{Cr}$ & $\mathrm{Pb}$ & $\mathrm{Ni}$ & $\mathrm{Cd}$ & $\mathrm{Fe}$ & $\mathrm{Mn}$ & $\mathrm{Zn}$ & $\mathrm{Cu}$ \\
\hline & -- & & & & $\mathrm{mg} \mathrm{L}$ & & & 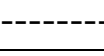 & - \\
\hline Água Potável & 3,0 & 0 & 0 & 0 & 0 & 0,12 & 0 & 0,01 & 0 \\
\hline Água Residuária & 5,5 & 0 & 0 & 0 & 0 & 0,74 & 0,01 & 0,03 & 0 \\
\hline
\end{tabular}

* Análise realizada no laboratório de qualidade da água do Departamento de Engenharia Rural - FCAV/UNESP-Jaboticabal - SP.

Durante a condução do experimento, foram realizadas as determinações de altura das plantas (distância da superfície do substrato à folha mais alta) e diâmetro do colmo (usando paquímetro digital, no centro dos colmos das plantas). Para ambas as medições, foram escolhidas 12 plantas, que representaram toda a parcela, aos 21; 42; 63 e 84 dias após a emergência das mesmas. Para o cálculo da irrigação, foi utilizado um atmômetro modificado, aparelho que, segundo BRONER \& LAW (1991), pode estimar a evapotranspiração de referência com boa precisão, cujas medidas de evaporação foram tomadas como base para definir as lâminas de água de irrigação. A irrigação inicial foi feita após os substratos estarem em capacidade de campo, considerando uma reposição diária de $100 \%$ da evapotranspiração, feita manualmente com regador.

\section{RESULTADOS E DISCUSSÃO}

Nas Tabelas 4 a 6, são apresentados os resultados da análise estatística dos valores de altura média da parte aérea das plantas (H) e o diâmetro do colmo (D). A análise estatística das variáveis estudadas demonstrou, por meio do teste $\mathrm{F}$, que os substratos apresentaram efeitos significativos na variação do desenvolvimento do ipê-amarelo.

Constatou-se que o substrato 1 (Tabela 4) esteve sempre entre os substratos que apresentaram os piores desempenhos no que diz respeito à altura média da parte aérea das plantas, fato este que talvez possa ser explicado pelas menores quantidades de nutrientes apresentadas pelo mesmo (Tabela 2).

Por intermédio da Tabela 4, pode-se constatar, ainda, a ocorrência da diferença entre os resultados apresentados para a altura média de plantas ao utilizarem-se diferentes fontes de água para irrigação (água potável x residuária), na qual as plantas onde se utilizou água residuária, apresentaram os menores valores de altura aos 21 dias após emergência, ocorrendo inversão deste comportamento quando comparado à água potável nas demais observações (42; 63 e 84 dias após emergência). Tal fato talvez possa ser explicado pela menor dependência nutricional advinda de fontes externas a plantas logo após a germinação, uma vez que estas obtêm estes nutrientes da própria semente na fase inicial de desenvolvimento, concordando com LUCENA et al. (2006) e SOARES et al. (2002); porém, ao cessarem estas reservas com o passar do tempo, a maior quantidade de nutrientes presentes na água residuária possa ter feito a diferença. 
TABELA 4. Altura média da parte aérea $(\mathrm{H})$ das plantas $(\mathrm{cm})$ aos $21 ; 42 ; 63$ e 84 dias após a emergência nos substratos estudados. Average height $(\mathbf{H})$ of the shoots $(\mathbf{c m})$ at 21, 42, 63 and 84 days after emergency in the substrates studied.

\begin{tabular}{|c|c|c|c|c|}
\hline \multirow[b]{2}{*}{ Substratos (S) } & \multicolumn{4}{|c|}{ Altura Média das Plantas } \\
\hline & 21dias & 42 dias & 63 dias & 84 dias \\
\hline 1 & $2,73 \mathrm{~b}$ & $7,61 \mathrm{~b}$ & $13,25 \mathrm{c}$ & $18,25 \mathrm{c}$ \\
\hline 2 & $2,98 \mathrm{a}$ & $8,44 \mathrm{a}$ & $13,63 \mathrm{ab}$ & $18,64 \mathrm{~b}$ \\
\hline 3 & $2,65 \mathrm{~b}$ & $8,32 \mathrm{a}$ & $13,71 \mathrm{ab}$ & $18,83 \mathrm{~b}$ \\
\hline 4 & $2,75 \mathrm{ab}$ & $8,26 \mathrm{a}$ & $13,60 \mathrm{~b}$ & $19,33 \mathrm{a}$ \\
\hline 5 & $2,67 \mathrm{~b}$ & $8,41 \mathrm{a}$ & $13,73 \mathrm{ab}$ & $19,55 \mathrm{a}$ \\
\hline 6 & $2,76 \mathrm{ab}$ & $8,48 \mathrm{a}$ & $13,75 \mathrm{ab}$ & $18,75 \mathrm{~b}$ \\
\hline 7 & $2,83 \mathrm{ab}$ & $8,25 \mathrm{a}$ & $13,93 \mathrm{a}$ & $18,95 \mathrm{~b}$ \\
\hline 8 & $2,59 \mathrm{~b}$ & $8,23 \mathrm{a}$ & $13,56 \mathrm{bc}$ & $18,65 \mathrm{~b}$ \\
\hline Teste $(\mathrm{F})$ & $2,78^{*}$ & $5,47 * *$ & $7,34 * *$ & $16,03 * *$ \\
\hline \multicolumn{5}{|l|}{ Água (A) } \\
\hline Potável (1) & $2,81 \mathrm{a}$ & $8,04 \mathrm{~b}$ & $13,48 \mathrm{~b}$ & $18,67 \mathrm{~b}$ \\
\hline Residuária (2) & $2,68 \mathrm{~b}$ & $8,46 \mathrm{a}$ & $13,81 \mathrm{a}$ & $19,66 \mathrm{a}$ \\
\hline Teste $(\mathrm{F})$ & $10,40 * *$ & $33,92 * *$ & $45,61 * *$ & $49,23 * *$ \\
\hline $\mathrm{S} \times \mathrm{A}$ & $1,89 \mathrm{NS}$ & $3,61^{*}$ & $6,07 * *$ & $13,51 * *$ \\
\hline C.V. & 5,75 & 3,44 & 1,45 & 1,20 \\
\hline
\end{tabular}

Ainda pela Tabela 4, nota-se a interação existente entre os substratos e as águas utilizadas na irrigação, do $42^{\underline{0}}$ dia de avaliação, sendo possível afirmar que os mesmos agem de forma conjunta sobre o crescimento das plantas e, sendo assim, tabelas de desdobramento foram elaboradas para melhor visualização destes resultados.

TABELA 5. Desdobramento da interação substrato x água para altura da parte aérea $(\mathrm{cm})$, aos 42 ; 63 e 84 dias após emergência. Deployment of interaction substrate $\mathbf{x}$ water to the height of the shoots $(\mathrm{cm})$ at 42,63 and 84 days after emergency.

\begin{tabular}{cllllll}
\hline \multirow{2}{*}{ Substratos } & \multicolumn{3}{c}{ Água Potável } & \multicolumn{3}{c}{ Água Residuária } \\
\cline { 2 - 7 } & 42 dias & 63 dias & 84 dias & 42 dias & 63 dias & 84 dias \\
\hline 1 & $7,25 \mathrm{~d}$ & $12,81 \mathrm{~b}$ & $17,75 \mathrm{~d}$ & $7,98 \mathrm{a}$ & $13,69 \mathrm{~b}$ & $18,75 \mathrm{~d}$ \\
2 & $8,25 \mathrm{ab}$ & $13,70 \mathrm{a}$ & $18,65 \mathrm{bc}$ & $8,63 \mathrm{a}$ & $13,56 \mathrm{~b}$ & $18,63 \mathrm{~d}$ \\
3 & $8,37 \mathrm{ab}$ & $13,68 \mathrm{a}$ & $18,73 \mathrm{bc}$ & $8,28 \mathrm{a}$ & $13,74 \mathrm{~b}$ & $18,93 \mathrm{~cd}$ \\
4 & $8,08 \mathrm{abc}$ & $13,36 \mathrm{a}$ & $18,73 \mathrm{bc}$ & $8,45 \mathrm{a}$ & $13,84 \mathrm{~b}$ & $19,93 \mathrm{a}$ \\
5 & $8,30 \mathrm{ab}$ & $13,57 \mathrm{a}$ & $19,65 \mathrm{a}$ & $8,53 \mathrm{a}$ & $13,89 \mathrm{ab}$ & $19,45 \mathrm{ab}$ \\
6 & $8,48 \mathrm{a}$ & $13,67 \mathrm{a}$ & $18,95 \mathrm{~b}$ & $8,48 \mathrm{a}$ & $13,84 \mathrm{~b}$ & $18,55 \mathrm{~d}$ \\
7 & $7,73 \mathrm{~cd}$ & $13,57 \mathrm{a}$ & $18,55 \mathrm{bc}$ & $8,78 \mathrm{a}$ & $14,30 \mathrm{a}$ & $19,35 \mathrm{bc}$ \\
8 & $7,90 \mathrm{bc}$ & $13,47 \mathrm{a}$ & $18,35 \mathrm{c}$ & $8,55 \mathrm{a}$ & $13,65 \mathrm{~b}$ & $18,95 \mathrm{~cd}$ \\
\hline Média & $8,04 \mathrm{~B}$ & $13,48 \mathrm{~B}$ & $18,67 \mathrm{~B}$ & $8,46 \mathrm{~A}$ & $13,81 \mathrm{~A}$ & $19,66 \mathrm{~A}$ \\
\hline
\end{tabular}

Médias seguidas de mesma letra minúsculas nas colunas e maiúsculas nas linhas não diferem entre si, pelo teste de Tukey, a $5 \%$ de probabilidade.

Ao avaliar a Tabela 5, aos 42; 63 e 84 dias, é possível notar que o desenvolvimento das plantas apresentou comportamento semelhante, ou seja, o substrato 1 foi o que apresentou os menores valores para o tamanho das plantas ao utilizar-se de água potável como irrigação; ao utilizar água residuária, o comportamento foi observado com menor intensidade, uma vez que o substrato 1 se mostrou semelhante a outros. Tal resultado talvez possa ter ocorrido devido a um maior suprimento nutricional, promovido pela água residuária, uma vez que, como observado pelas 
Tabelas 2 e 3, a disponibilidade de nutrientes promovida pelo substrato 1 é menor que os demais, e a maior disponibilidade destes nutrientes disponíveis na água residuária, respectivamente, supriu esta deficiência.

Em todos os dias de amostragem $(42 ; 63$ e 84) as médias de altura apresentadas nos tratamentos de água residuária foram superiores às apresentadas pela água potável, possivelmente pela maior quantidade de nutrientes presentes na primeira (Tabela 3).

Avaliando a Tabela 6, observa-se que, assim como ocorrido para altura média de plantas, o diâmetro do caule também obteve os menores valores para as plantas semeadas no substrato 1 aos 42 dias após emergência, e, ainda, este esteve entre os substratos que apresentaram os menores valores para esta variável, aos 63 e 84 dias, talvez também pelos mesmos motivos supostos para explicar a altura média das plantas.

NOBILE et al. (2007), estudando a altura de plantas de crisântemo em substratos com diferentes concentrações de composto de lixo urbano e irrigado com água residuária, observaram que concentrações de composto de lixo maiores que $30 \%$ influenciavam negativamente no desenvolvimento das plantas.

TABELA 6. Diâmetro de colmo(D) das plantas (mm) aos 42; 63 e 84 dias após a emergência nos substratos estudados. Stem diameter (D) of the plants $(\mathrm{mm})$ at 42,63 and 84 days after emergency in the substrates studied.

\begin{tabular}{|c|c|c|c|}
\hline \multirow{2}{*}{ Substrato (S) } & \multicolumn{3}{|c|}{ Diâmetro de colmo } \\
\hline & 42 dias & 63 dias & 84 dias \\
\hline 1 & $6,10 \mathrm{e}$ & $11,08 \mathrm{~d}$ & $19,13 \mathrm{c}$ \\
\hline 2 & $6,25 \mathrm{bc}$ & $11,11 \mathrm{~cd}$ & $19,69 \mathrm{a}$ \\
\hline 3 & $6,16 \mathrm{~d}$ & $11,14 \mathrm{bcd}$ & $19,38 \mathrm{bc}$ \\
\hline 4 & $6,20 \mathrm{~cd}$ & $11,26 \mathrm{a}$ & $19,75 \mathrm{a}$ \\
\hline 5 & $6,17 \mathrm{~d}$ & $11,15 \mathrm{bcd}$ & $19,75 \mathrm{a}$ \\
\hline 6 & $6,27 \mathrm{ab}$ & $11,23 \mathrm{a}$ & $19,60 \mathrm{ab}$ \\
\hline 7 & $6,32 \mathrm{a}$ & $11,16 \mathrm{bc}$ & $19,69 \mathrm{a}$ \\
\hline 8 & $6,21 \mathrm{bcd}$ & $11,20 \mathrm{ab}$ & $19,66 \mathrm{ab}$ \\
\hline Teste $(\mathrm{F})$ & $11,69 * *$ & $9,68 * *$ & $5,76 * *$ \\
\hline \multicolumn{4}{|l|}{ Água (A) } \\
\hline Potável (1) & $6,21 \mathrm{a}$ & $11,13 \mathrm{~b}$ & $19,49 \mathrm{~b}$ \\
\hline Residuária (2) & $6,21 \mathrm{a}$ & $11,21 \mathrm{a}$ & $19,67 \mathrm{a}$ \\
\hline Teste $(\mathrm{F})$ & $0,38 \mathrm{NS}$ & $50,32 * *$ & $13,43 * *$ \\
\hline $\mathrm{S} \times \mathrm{A}$ & $7,84 * *$ & $7,23 * *$ & $2,74 *$ \\
\hline C.V. & 0,62 & 0,40 & 0,99 \\
\hline
\end{tabular}

Médias seguidas de mesma letra na coluna não diferem entre si, a 5\% de probabilidade, pelo teste de Tukey. C.V., SxA e NS representam, respectivamente: coeficiente de variação, interação entre substratos e águas de irrigação e não significativo.*,** Significativo a $5 \%$ e $1 \%$ de probabilidade, respectivamente. \# Avaliação aos 21 dias não foi realizada devido os colmo das plantas estarem sensíveis ao paquímetro digital.

Pela mesma tabela, é percebida a interação existente entre o substrato e a fonte de água de irrigação utilizada no que diz respeito ao diâmetro do caule, podendo ser mais bem visualizado no desdobramento (Tabela 7).

Na Tabela 7 (aos 42 dias), observa-se que, para a irrigação feita com água potável, os substratos 1; 3 e 5 apresentaram valores inferiores aos apresentados pelos substratos 4; 6 e 7 . Já com a utilização de água residuária, o substrato 7 mostrou-se superior a todos os outros, com exceção do substrato 2, sendo que estes são os substratos que apresentam a maior quantidade de lixo orgânico em sua composição (60\% para o substrato 7 e $100 \%$ para o substrato 2) (Tabela 1). 
Com relação à água de irrigação utilizada, para os valores mensurados aos 42 dias após a emergência, não foi observada diferença significativa.

TABELA 7. Desdobramento da interação entre tratamento $\mathrm{x}$ água para diâmetro de colmo (mm) aos 42; 63 e 84 dias após emergência. Deployment of interaction between treatment $\mathbf{x}$ water for stem diameter $(\mathrm{mm})$ at 42,63 and 84 days after emergency.

\begin{tabular}{clcllll}
\hline \multirow{2}{*}{ Substratos } & \multicolumn{3}{c}{ Água Potável } & \multicolumn{3}{c}{ Água Residuária } \\
\cline { 2 - 7 } & $42 \mathrm{dias}$ & 63 dias & 84 dias & 42 dias & 63 dias & 84 dias \\
\hline 1 & $6,05 \mathrm{~d}$ & $11,05 \mathrm{~b}$ & $18,98 \mathrm{~b}$ & $6,14 \mathrm{c}$ & $11,12 \mathrm{c}$ & $19,28 \mathrm{c}$ \\
2 & $6,22 \mathrm{bc}$ & $11,10 \mathrm{~b}$ & $19,63 \mathrm{a}$ & $6,29 \mathrm{ab}$ & $11,13 \mathrm{c}$ & $19,75 \mathrm{abc}$ \\
3 & $6,15 \mathrm{c}$ & $11,15 \mathrm{ab}$ & $19,43 \mathrm{a}$ & $6,18 \mathrm{c}$ & $11,13 \mathrm{c}$ & $19,33 \mathrm{bc}$ \\
4 & $6,24 \mathrm{ab}$ & $11,25 \mathrm{a}$ & $19,58 \mathrm{a}$ & $6,15 \mathrm{c}$ & $11,27 \mathrm{a}$ & $19,93 \mathrm{a}$ \\
5 & $6,14 \mathrm{~cd}$ & $11,14 \mathrm{ab}$ & $19,60 \mathrm{a}$ & $6,19 \mathrm{c}$ & $11,16 \mathrm{bc}$ & $19,90 \mathrm{a}$ \\
6 & $6,32 \mathrm{a}$ & $11,13 \mathrm{~b}$ & $19,40 \mathrm{ab}$ & $6,21 \mathrm{bc}$ & $11,34 \mathrm{a}$ & $19,80 \mathrm{ab}$ \\
7 & $6,31 \mathrm{ab}$ & $11,06 \mathrm{~b}$ & $19,55 \mathrm{a}$ & $6,33 \mathrm{a}$ & $11,25 \mathrm{ab}$ & $19,83 \mathrm{a}$ \\
8 & $6,22 \mathrm{bc}$ & $11,14 \mathrm{ab}$ & $19,78 \mathrm{a}$ & $6,19 \mathrm{c}$ & $11,27 \mathrm{a}$ & $19,55 \mathrm{abc}$ \\
\hline Média & $6,21 \mathrm{~A}$ & $11,13 \mathrm{~B}$ & $19,49 \mathrm{~B}$ & $6,21 \mathrm{~A}$ & $11,21 \mathrm{~A}$ & $19,67 \mathrm{~A}$ \\
\hline
\end{tabular}

Médias seguidas de mesma letra minúscula (na coluna) e maiúscula (na linha) não diferem entre si, pelo teste de Tukey, a 5\% de probabilidade.

$\mathrm{Na}$ avaliação feita aos 63 dias (Tabela 7), foi notada superioridade de valores favorável ao substrato 4, em relação aos substratos $1 ; 2 ; 6$ e 7 ao utilizar para irrigação a água potável e ao utilizar-se de água residuária o mesmo substrato, assim como os de número 6 e 8 mostraram-se superiores aos de número $1 ; 2 ; 3$ e 5 . Nesta avaliação, fez-se presente a diferença entre as águas utilizadas no que se refere ao diâmetro do caule, sendo os valores apresentados pela água residuária superiores aos apresentados pelos da água potável (11,21 x 11,13mm, respectivamente).

Ao observar os valores dos diâmetros dos caules das plantas aos 84 dias (Tabela 7), nota-se que para a irrigação feita com água potável, o substrato 1 foi semelhante apenas ao substrato 6, sendo inferiores a todos os demais. Para a irrigação feita com água residuária, o substrato 1 apresentou valores de diâmetro de caule inferiores aos observados para os substratos 4; 5; 6 e 7 .

Com relação às águas utilizadas, novamente a água residuária apresentou os maiores valores médios $(19,67 \mathrm{~mm})$ quando comparados aos valores médios apresentados pela água potável $(19,49 \mathrm{~mm})$.

De acordo com NOBILE et al. (2006), a qualidade da água (potável ou residuária) não influenciou no diâmetro do caule de mudas cítricas, mas pode observar diferença significativa com o uso de composto de lixo, sendo o maior diâmetro de caule obtido com o substrato formado de $60 \%$ de casca de pínus e $40 \%$ de lixo orgânico.

\section{CONCLUSÕES}

Os substratos estudados apresentaram diferença para altura média das plantas e diâmetro de colmo em todos os períodos de avaliação.

As águas utilizadas para irrigação apresentaram diferença significativa para altura média de plantas em todo o período de análise e aos 63 e 84 dias para o diâmetro do colmo, sendo que, na média, a água residuária apresentou os melhores resultados.

Os substratos 4 e 5 foram os que apresentaram os melhores resultados no desenvolvimento das mudas de ipê-amarelo. 


\section{REFERÊNCIAS}

AUGUSTO, D.C.C.; GUERRINI, I.A.; ENGEL, V.L.; ROUSSEAU, G.X. Utilização de esgotos domésticos tratados através de um sistema biológico na produção de mudas de Croton floribundus Spreng. (Capixingui) e Copaifera langsdorffii Desf. (Copaíba). Revista Árvore, Viçosa-MG, v.27, n.3, p.335-342, 2003.

BENITO, M.; MASAGUER, A.; MOLINER, A.; ARRIGO, N.; PALMA, R.; EFFRON, D. Evaluation of maturity and stability of pruning waste compost and their effect on carbon and nitrogen mineralization in soil. Soil Science, Madrid, v.170, n.5, p.360-370, 2005.

BRONER, I.; LAW, R.A.P. Evaluation of modified atmometer for estimating reference ET. Irrigations Science, New York, v.12, p.21-26, 1991.

CROMER, R.N. Irrigation of radiata pine with wastewater: A review of the potential for tree growth and water renovation. Australian Forest Research, Canberra, v.43, p.87-100, 1980.

GROSSI, M.G.L. Avaliação da qualidade dos produtos obtidos de usinas de compostagem brasileiras de lixo doméstico através de determinação de metais pesados e substâncias orgânicas tóxicas. 1993. 222 f. Tese (Doutorado) - Universidade de São Paulo, São Paulo, 1993.

HACH Company. Spectrophotometer Instrument Manual. Loveland. Colorado/USA, 1996.

IBGE. INSTITUTO BRASILEIRO DE GEOGRAFIA E ESTATÍSTICA. STATCART - Base de informações por setor censitário, Estância de Águas de São Pedro- SP - Censo Demográfico 2000. Rio de Janeiro: 2002. CD-ROM.

LABORATÓRIO NACIONAL DE REFERÊNCIA VEGETAL - Lanarv. Análise de corretivos, fertilizantes e inoculantes: métodos oficiais do Laboratório Nacional de Referência Vegetal. Brasília: LANARV, 1988. 104 p.

LUCENA, A.M.A.; ALMEIDA, F.A.C.; COSTA, F.X.; GUERRA, H.O. Emprego de substratos irrigados com água de abastecimento e residuária na propagação do flamboyant. Revista de Biologia e Ciência da Terra, João Pessoa, v.6, n.1, p.115-121, 2006.

NOBILE, F O.; GALBIATTI, J.A.; CORDIDO, J.P.B.R.; ANDRIÃO, M.A.; MURAISHI, R.I. Avaliação de níveis de irrigação e a utilização de composto de lixo orgânico na formação de mudas cítricas. In: CONGRESSO BRASILEIRO DE ENGENHARIA AGRÍCOLA, 35., 2006, João Pessoa. Anais.... João Pessoa: Agroenergia, 2006. 1 CD-ROM.

NOBILE, F.O.; GALBIATTI, J.A.; MURAISHI, R.I. ; CORDIDO, J.P.B.R.; ANDRIÃO, M.A. Doses de composto de lixo no substrato e dois níveis de irrigação em crisântemo. In: CONGRESSO BRASILEIRO DE ENGENHARIA AGRÍCOLA, 37., 2007, Bonito. Anais... Jaboticabal: Associação Brasileira de Engenharia Agrícola, 2007. 1 CD-ROM.

OLIVEIRA, F.C.; MATTIAZZO, M.E.; MARCIANO, C.R.; ABREU JUNIOR, C.H.Alterações em atributos químicos de um latossolo pela aplicação de composto de lixo urbano. Pesquisa Agropecuária Brasileira, Brasília, v.37, n.12, p.529-538, 2002.

SANDERSON, K.C. Use of sewage-refuse compost in the production of ornamental plants. HortSciense, Alexandria, v.15, n.2, p.173-178, 1980.

SOARES, F.A.L.; GHEYI, H.R.; VAN HAANDEL, A.C.; BELTRÃO, N.E. de M. Viabilidade do uso de lodo e água de esgoto no cultivo de girassol: germinação e vigor inicial. In: CONGRESSO BRASILEIRO DE ENGENHARIA AGRÍCOLA, 31., 2002, Salvador, Anais... Jaboticabal: Associação Brasileira de Engenharia Agrícola, 2002. 1 CD-ROM. 